\title{
Antihyperlipidemic and antiatherogenic activity of simvastatin may involve modulation of the expression of lecithin:cholesterol acyl transferase
}

\author{
Adeniran Sanmi Adekunle ${ }^{凶}$, John Olabode Fatoki and Temitope Isaac Adelusi \\ Department of Biochemistry, Ladoke Akintola University of Technology, Nigeria
}

Introduction: The statin-induced effects on high density lipoprotein (HDL) are relatively small compared with those of low density lipoprotein (LDL) and, as a result, most clinical trials of statins are underpowered with respect to HDL parameters. This study experimentally investigated, the effects of statin on serum lipids, atherogenic index and examined the possibility of a relationship amongst serum concentrations of HDL-C, atherogenic index and activity of lecithin:cholesterol acyl transferase. Method: Thirty albino rats equally divided into 2 groups were used for the study. Group 1 was given $0.05 \mathrm{mg} / \mathrm{g}$ of statin daily for 28 days, while group 2 served as control. HDL concentration was determined as a measure of HDL-C. Total cholesterol (TC), triglyceride (TG) and HDL-C were determined spectrophotometrically while LDL-C was calculated using the Frieldwald formula. Effect on the activity of lecithin:cholesterol acyl transferase was determined by the difference between the amount of free cholesterol converted to cholesteryl ester in the two experimental groups. Effects on body and relative organs weights were also determined. Results: The administration of statin caused a significant increase in serum concentration of HDL-C, while levels of LDL-C, triglyceride and total cholesterol were reduced. Simvastatin caused a significant reduction in the atherogenic index (TC/HDL-C; LDLC/HDL-C). The administration of statin significantly induced the activity of lecithin:cholesterol acyl transferase (LCAT) as evident by reduced serum concentration of free cholesterol when compared with control. The administration of statin caused reduced body and relative organs weights. Conclusion: The study showed that serum antihyperlipidemic and antiatherogenic activity of statin may involve the induction of LCAT.

Key words: atherogenicity, statin, lipids, lipoproteins, lecithin :cholesterol acyl transferase

Received: 09 May, 2013; revised: 02 September, 2013; accepted: 19 September, 2013; availabe on-line: 22 November, 2013

\section{INTRODUCTION}

The use of statins has revolutionized the management of people at risk of having a cardiovascular event. Several effects of statins have the potential to reduce cardiovascular risk, with compelling evidence that the statin-induced reductions in LDL cholesterol (LDL-C) are implicated (Baigent et al., 2005; Sipahi et al., 2006) Currently, six different statins (simvastatin,pravastatin, lovastatin, fluvastatin, rosuvastatin and atorvastatin) are approved for treatment of hypercholesterolemia in hu- mans (Brown, 2001). Despite differences in their pharmacokinetic profiles, all statins have at least one characteristic in common: they block the conversion of HMGCoA to mevalonic acid with consecutive attenuation of the biosynthesis of cholesterol, which is associated with a reduction in serum total and low-density lipoprotein (LDL) cholesterol of as much as $20-31 \%$ and $28-42 \%$ during chronic treatment (Andrew et al., 2001). Because of these properties, statins have become the most widely prescribed lipid-lowering drugs to patients with elevated serum cholesterol levels. The discovery of statins led to important improvements in the primary and secondary prevention of coronary artery disease (CAD). Initial studies explored the impact of statin therapy on CAD progression and regression. Although the angiographic changes in response to therapy were modest, the accompanying clinical benefit appeared significant. Subsequent large prospective clinical trials have provided unequivocal evidence that cholesterol-lowering therapy with this class of compounds not only reduces the major coronary event rate in primary and secondary prevention, but also

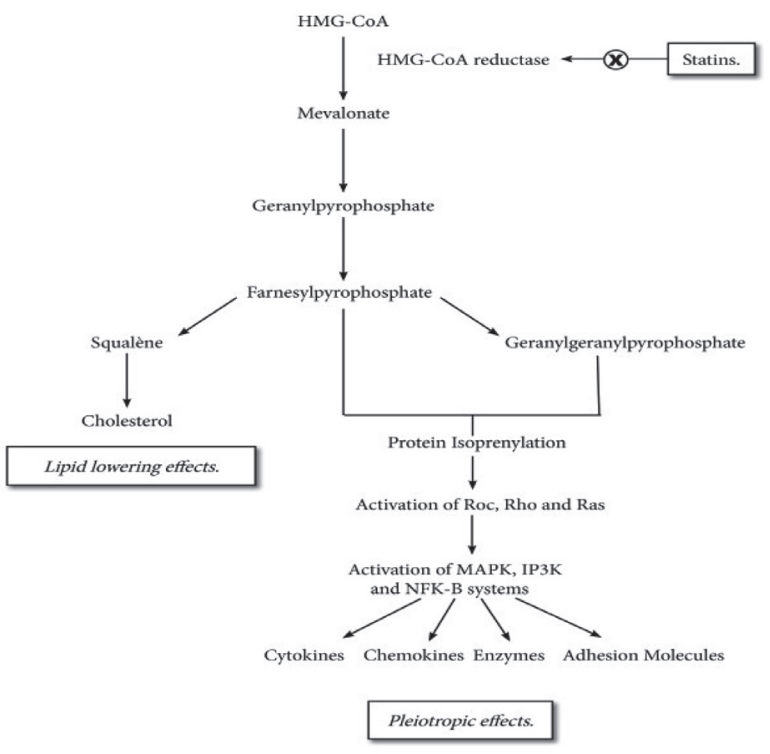

Scheme 1. Schematic diagram of how statins inhibit cholesterol biosynthesis (Loecker et al., 2012)

e-mail: kunleniran@yahoo.com

Abbreviations: CAD, coronary artery disease; CETP, cholesteryl ester transfer protein; LDL, low-densitylipoprotein; VLDL, very-lowdensitylipoprotein 
reduces all-cause mortality in secondary prevention. Several large trials demonstrated that statins are not only safe and well-tolerated but also significantly decrease the cardiovascular morbidity and mortality in hypercholesterolemic patients in both primary and secondary prevention (Shepherd et al., 1995; Sacks et al., 1996; Down et al., 1998). However, the striking benefit achieved with statin treatment in patients with a wide range of cholesterol levels, which cannot be attributed to their cholesterol lowering effect alone, has raised the question about the possible presence of additional effects of statins beyond their impact on serum cholesterol levels. Indeed, in recent years a substantial quantity of data has accumulated a notion that statins exert various effects on multiple targets. Studies have shown that statin inhibits the biosynthesis of isoprenoids which are essential for the posttranslational modification of several proteins involved in important intracellular signaling pathways (e.g. small GTP-binding proteins Ras and Rho). Another pathway affected by statins seems to be the regulation of the activity of the enzyme cholesteryl ester transfer protein (CETP), which transfers cholesteryl ester to verylow-densitylipoprotein (VLDL) and LDL (Bruce \& Tall, 1995). This study thus sought to evaluate the regulation of lecithin:cholesterol acyl transferase by simvastatin in experimental animal model.

\section{METHODS}

Materials. Simvastatin drug was purchased from a pharmacy in Ogbomoso, while the kits for measurement of lipids and lipoproteins were LABKIT products of CHEMELEX, SA (Barcelona, Spain). All other chemicals used including solvents are of analytical grade.

Study design. Thirty adult Wistar rats with average weight of 190 grams were purchased and housed in wellventilated wire cages to acclimatize for few days. They were fed with regular pelleted animal feed and were given unrestricted access to clean water. The protocol conforms to the guidelines of the National Institute of Health (NIH) (NIH publication 85-23, 1985) for laboratory animal care and use. Animals were randomly divided into two groups of fifteen animals each. Rats in group 1 were orally administered simvastatin $(0.05 \mathrm{mg} / \mathrm{g})$ per rat per day for 28 consecutive days. Group 2 served as control (received drug-vehicle, water). On day 29, the rats were sacrificed by cervical dislocation. The rats were made to fast for 10-12 hours overnight and fasting blood samples were collected through cardiac puncture and then centrifuged at $1500 \times \mathrm{g} / \mathrm{min}$ for $10 \mathrm{~min}$ to obtain serum and stored at $-20^{\circ} \mathrm{C}$ till further analysis was done.

Determination of biochemical parameters. Analyses of lipids and lipoprotein. Total cholesterol, triglyceride, and HDL-C were analyzed using spectrophotometric methods, while LDL-C was calculated. Total cholesterol concentration in the serum was determined spectrophotometrically using the cholesterol oxidase method at $546 \mathrm{~nm}, 37^{\circ} \mathrm{C}$ (Allain et al., 1974). HDL-C was determined by the spectrophotometric method of (Assman et al., 1983) at $500 \mathrm{~nm}, 37^{\circ} \mathrm{C}$. Triglycerides were determined using the spectrophotometric method (Buccolo \& David, 1973). Low density lipoprotein cholesterol was calculated using the Friedwald formula (Friedwald et al., 1972).

Estimation of atherogenicity. The atherogenic index was estimated by calculating TC: HDL-C and LDL-C: HDL-C ratios.
Lecithin: cholesterol acyl transferase assay method. The activity of LCAT was assayed in serum using the method of Hitz et al., (1983). Briefly, a pool of serum was heated at $56^{\circ} \mathrm{C}$ for 30 minutes to inactivate the LCAT. The inactivated serum was incubated at $4^{\circ} \mathrm{C}$ for 15 minutes with $0.2 \%$ dextran sulfate, which caused the elimination of two-thirds of the lipoproteins (LDL+VLDL). This was sedimented by centrifugation at $1750 \times g$ for 15 minutes. The supernatant containing HDL was used as the substrate. A sample of the substrate $(0.6 \mathrm{ml})$ was mixed with $1.0 \mathrm{ml}$ of isopropyl alcohol while the remaining mixture was incubated at $27^{\circ} \mathrm{C}$ for 90 minutes. The precipitate was removed by centrifugation and the supernatant was taken for the estimation of free cholesterol present in the test sample at zero time. After 90 minutes, a $0.2 \mathrm{ml}$ sample of the incubated mixture was mixed with $1.0 \mathrm{ml}$ of isopropyl alcohol and the remaining mixture was incubated at $27^{\circ} \mathrm{C}$ for a further 90 minutes. At the end of 180 th minutes, $0.2 \mathrm{ml}$ of the incubated mixture was treated with $1.0 \mathrm{ml}$ of isopropyl alcohol to arrest the reaction. The precipitated protein in all of the tubes were separated by centrifugation and the free cholesterol content in the supernatant was estimated. Control tubes containing only the substrate were treated similarly to check for the complete inactivation of serum during substrate preparations. LCAT activity was expressed as a function of the disappearance of free cholesterol during the incubation period.

Statistical analysis. Values were expressed as mean \pm S.D. (standard deviation. Differences between groups were statistically compared using the students't-test. The results were considered statistically significant for $p<0.05$.

\section{RESULTS}

Table 1 depicts the effects of simvastatin on serum lipid profile. The administration of simvastatin caused a significant increase and decrease in serum concentrations of HDL-C and free cholesterol respectively ( $p>0.05)$. Though not statistically significant, simvastatin reduced serum concentrations of total cholesterol, triglyceride and LDL-C. Simvastatin caused a significant reduction in the atherogenic index (TC: HDL-C and LDL-C: HDL-C ratios) $(p>0.05)$. Table 2 shows the effects of the administration of simvastatin on body and organs weights. Simvastatin did not cause any appreciable reduction in the body and organs weight $(p<0.05)$.

Table 1. Effects of statin on serum concentrations of total cholesterol, triglyceride, HDL-C, LDL-C and the activity of lecithin:cholesterol acyl transferase in albino rats.

\begin{tabular}{lcc}
\hline Lipid Parameters & $\begin{array}{c}\text { Group 1 } \\
(\mathrm{M} \pm \text { S.D. })\end{array}$ & $\begin{array}{c}\text { Group 2 } \\
(\mathrm{M} \pm \text { S.D. })\end{array}$ \\
\hline Total cholesterol $(\mathrm{mg} / \mathrm{dl})$ & $1.84 \pm 0.31^{\mathrm{a}}$ & $1.98 \pm 0.48^{\mathrm{a}}$ \\
\hline Triglyceride $(\mathrm{mg} / \mathrm{dl})$ & $0.86 \pm 0.25^{\mathrm{b}}$ & $0.96 \pm 0.40^{\mathrm{b}}$ \\
\hline $\mathrm{HDL}-\mathrm{C}(\mathrm{mg} / \mathrm{dl})$ & $1.33 \pm 0.19^{\mathrm{a}}$ & $1.10 \pm 0.12^{\mathrm{b}}$ \\
\hline $\mathrm{LDL}-\mathrm{C}(\mathrm{mg} / \mathrm{dl})$ & $0.25 \pm .17^{\mathrm{a}}$ & $0.45 \pm 0.51^{\mathrm{a}}$ \\
\hline $\mathrm{TC} / \mathrm{HDL}-\mathrm{C}$ & $1.36 \pm 0.12^{\mathrm{a}}$ & $1.72 \pm 0.30^{\mathrm{b}}$ \\
\hline LDL-C/HDL-C & $0.17 \pm 0.03^{\mathrm{a}}$ & $0.38 \pm 0.21^{\mathrm{b}}$ \\
\hline $\begin{array}{l}\text { Free cholesterol (index of LCAT } \\
\text { activity) }(\mathrm{mg} / \mathrm{dl})\end{array}$ & $0.06 \pm 0.05^{\mathrm{a}}$ & $0.18 \pm 0.08^{\mathrm{b}}$ \\
\hline
\end{tabular}

Values are means \pm S.D.; $n=15$. Values bearing different alphabets in the same row are significantly different $(p<0.05)$.Group 1: animals given therapeutic dose of statin; Group 2: animals that were not given statin i.e. control. 
Table 2. Effect of the administration of simvastatin on body and visceral organs weights in rats.

\begin{tabular}{lllll}
\hline Groups & body weight gain $(\mathrm{g})$ & weight of liver $(\mathrm{g})$ & weight of heart $(\mathrm{g})$ & weight of kidney(g) \\
\hline Group 1 & $45.13 \pm 3.05 \mathrm{a}$ & $2.41 \pm 0.11 \mathrm{a}$ & $0.38 \pm 0.02 \mathrm{a}$ & $0.45 \pm 0.01 \mathrm{a}$ \\
\hline Group 2(control) & $60.20 \pm 1.64 \mathrm{~b}$ & $2.85 \pm 0.14 \mathrm{a}$ & $0.41 \pm 0.07 \mathrm{a}$ & $0.49 \pm 0.01 \mathrm{a}$ \\
\hline
\end{tabular}

Values are means $\pm S D ; n=15$. Values bearing different alphabets in the same column are significantly different ( $p<0.05)$.Group 1 : animals given therapeutic dose of statin; Group 2: animals that were not given statin i.e. control.

\section{DISCUSSION}

Hypercholesterolemia is a known and significant risk factors for the development of coronary heart disease (CHD) and death (Masumori et al., 2002). The epidemiological study established that a high concentration of serum cholesterol confers a high risk of CHD. Although, the use of statin in the management of hypercholesterolemia had been demonstrated as first-line pharmacotherapeutic agents, the effects of statin on atherosclerosis and lipid levels in animal models are very paradoxical (Guohua et al., 2011). While Bea et al., 2002; Wang et al., 2002 and Choudhury, et al., 2004 and showed that the administration of simvastatin to apoE-deficient mice caused a significant elevation of plasma cholesterol, Masunori et al., (2002) demonstrated that simvastatin caused a reduction in plasma concentration of total cholesterol. Our data was in agreement with Guohua et al., 2011 who reported that the administration of simvastatin to apoE-deficient mice fed a high cholesterol diet caused a reduction in plasma total cholesterol levels.

In this study, HDL-C concentration was increased by the administration of simvastatin. One of the objectives of this study was to determine and compare the concentrations of HDL-C in rats administered simvastatin and healthy controls and to investigate the relationship between possible changes in serum HDL-C concentration and the activity of lecithin:cholesterol acyl transferase. The administration of simvastatin significantly increased concentration of HDL-C. Although, previous studies reported the ability of statins to increase the concentration of HDL-C (Jones et al, 1998; Jones et al., 2003; Assman and Gotto, 2004; Shubhangi et al, 2013), findings here further affirmed this capacity. A risk factor for the cardiovascular disease includes a low serum level of HDLcholesterolol. Low level of HDL-cholesterol has been linked to an increased risk of CVD through series of epidemiological and clinical studies (Barter et al., 2007). HDL-C is actively involved in reverse cholesterol transport; hence its low level plays a direct role in the atherogenic process. Raising HDL-C is widely encouraged as a means of reducing the predisposition to atherogenesis. In this study, treatment with simvastatin led to a significant elevation of plasma HDL-cholesterol, indicating its protective role against CVD. Studies have shown cardioprotective activities of statin administration (Gordon et al., 1977; Mackness et al., 2000).

HDL plays a prominent role in lipids metabolism. The inverse correlation between HDL levels and cardiovascular heart disease risk has been explained by the ability of HDL to remove cholesterol from the peripheral circulation and deliver it to the liver for excretion in the bile, in the process known as reverse cholesterol transport (Endo et al., 1977). Reduced serum concentration of high density lipoprotein, even in the presence of reduced low density lipoprotein, has been linked to the occurrence of cardiovascular disorder. Since the original seminal hypothesis of Gordon et al. was proposed in 1977
(Sposito and Chapman, 2002), the atheroprotective role associated with HDL has become widely recognized. A plethora of potential mechanisms that may account for the cardioprotective effects of HDL have been documented, several of which may be mutually interactive and, indeed, synergistic. ApoB100-containing particles deliver cholesterol to peripheral tissues and to the developing plaque, whereas HDL, primarily through the scavenger receptor class B type I (SR-BI/Cla-1) and LIMPII analogous 1 (CD-36) receptor on human macrophages, is able to pick up cholesterol from atherosclerotic plaque and return it to the liver for excretion in form of bile acids. Although reverse cholesterol transport is one of the major functions of HDL particles, HDL exerts several other potentially anti-atherogenic actions.

Simvastatin equally caused reduction in the mean serum concentrations of triglyceride, total cholesterol and LDL-C. In addition to decreasing LDL-C concentration through the inhibition of HMG-CoA reductase, the enzyme involved in the rate limiting step of cholesterol biosynthesis, statins lower triglycerides and modestly increase HDL-C (Matsuda et al., 1993). This has been further confirmed in this study as the mean value of HDL$\mathrm{C}$ and mean value of TG was observed to be moderately increased and slightly decreased respectively. An overall decrease was observed in the mean concentration on total cholesterol, triglyceride and LDL-C of the test group. This overall decrease could be as a result of the lipid lowering effect of simvastatin which inhibited the enzyme HMG-CoA reductase involved in the rate limiting step of cholesterol biosynthesis. This finding is similar to the report of (Galle et al., 1994). Rosenfeld et al., (1983), reported that in the atherogenic dyslipidaemias, statins act to decrease levels of atherogenic lipoproteins and to re-establish equilibrium between cardioprotective HDL and atherogenic ApoB-containing lipoproteins. As a consequence, cholesterol efflux from the plaque is enhanced, whereas cholesterol influx from atherogenic lipoproteins is considerably diminished. A decrease in plaque cholesterol and macrophage content decreases inflammation and enhances plaque stability, resulting in a decrease in cardiovascular events. This is in line with the decreased mean concentrations of total cholesterol, LDL-C and triglyceride and increased mean concentration of HDL-C observed in this study.

HDL has various species, identified on the basis of their major apolipoprotein (apo) components (apoA-I or apoA-II), density (HDL2 and HDL3) and electrophoretic mobility ( $\alpha$ and pre- $\beta$ ) (Vakeva et al., 1994). Changes in HDL levels more closely reflect variations in the HDL2 subfraction rather than HDL3. Several studies have shown that low levels of HDL2 and HDL3 are associated with increased progression of atherosclerosis and risk of cardiovascular disease (Fleisher et al., 1982). Since HDL and ApoA-I constitute major receptors of cholesterol in the cholesterol efflux, increasing HDL levels may increase cholesterol efflux and reverse cholesterol transport, contributing to reduced cardiovascular disease 
risks. Many attempts have been made to enhance HDL levels as anti-atherogenesis therapy (Bela et al., 2009) and one of such are statins which could decrease intracellular hepatic cholesterol and up-regulate LDL receptors (Francone \& Fielding, 1991) which remove both LDL and triglyceride-rich lipoproteins (VLDL and IDL) from the circulation, thereby reducing plasma LDL and triglyceride levels. In addition, statins could also raise HDL levels (Wang et al., 1997) which may be mediated through increase in the synthesis and secretion of apoA-I (Guohua et al., 2011). Several intervention studies in both primary and secondary prevention support the benefit of treating patients who have low HDL levels with a statin to decrease cardiovascular risk in primary and secondary prevention settings (Getz et al., 2009). All of these previous studies correspond with the significantly increased mean concentration of HDL-C obtained recorded when simvastatin was administered to rats in this study.

This study investigated the effect of simvastatin on rate of disappearance of free cholesterol (as an index of LCAT activity) and assessed any relationship between changes in serum concentrations of HDL-C and the rate of disappearance of free cholesterol. Results from this study showed simultaneous increase in the serum concentration of HDL-C and reduced free cholesterol level i.e. increased rate of disappearance of free cholesterol (increased LCAT activity). The implication is that the maturation of HDL particle is associated with enhanced LCAT activity causing reduced serum concentration of free cholesterol due to its increased rate of conversion to cholesteryl ester. However, the shortcoming is that cholesteryl ester was not measured to further support this observation. A fundamental key aspect of HDL metabolism, in terms of the ability of the particle to protect against atherosclerosis, is the recycling of apo A-I and the other HDL apolipoproteins between the lipid-free and the lipid-associated forms. This process is known as particle remodeling. This recycling delays the clearance of lipid-free apolipoproteins via the kidney and, thus, maintains HDL levels in the plasma (Nandagopal et al., 2008) Lecithin:cholesterol acyl transferase(LCAT), a $63 \mathrm{Kda}$ glycoprotein enzyme which is synthesized in the liver and secreted in the plasma (Packman et al., 1985; Zeiher et al., 1994) facilitates HDL recycling by converting discoidal particles into larger, spherical ones. As LCAT esterifies the free cholesterol in the HDL discs, the resulting cholesteryl esters are sequestered into the center of the particle; and the discs become spherical in shape. This remodeling depletes the particle surface of cholesterol and establishes a concentration gradient down which additional unesterified cholesterol moves from the cell membranes and other lipoproteins into the HDL fraction. In maintaining the gradient between the cholesterol content of the peripheral cell and HDL in plasma, LCAT plays a critical role in the initial steps of reverse cholesterol transport (Sugiyama et al., 1968). This study implied that simvastatin enhanced activity of lecithin:cholesterol acyl transferase.

Since studies have shown that simvasstatins lower the blood lipid level by inhibiting HMG CoA reductase causing reduced synthesis of cholesterol (Nofer et al., 1998) and it has also been established that LCAT catalyzes the esterification of cholesterol during the reverse cholesterol transport process (Curtis and Plow, 1984). This present study showed that cardioprotective property of simvastatin may not only be by inhibition of HMG CoA activity thereby reducing endogenous cholesterol biosynthesis, but may also include induction of LCAT activity thus promoting esterification of free cholesterol.
In this study, the body and organs weight in simvastatin administered rats were decreased significantly when compared with control animals. The hypolipidemic and antiatherogenic effects of simvastatin may be responsible for the beneficial action of this drug on body and organs weights. (Koller et al., 1982) reported similar findings with topiramate, atorvastatin per se and the combination of topiamate + atorvastatin in cafeteria diet fed animals significantly decreasing the weight of kidney, liver, spleen and heart.

\section{REFERENCES}

Allain CC, Poon LS, Chan CSG (1974) Enzymatic determination of total serum cholesterol. Clin Chem 20: 470-475.

Andrews TC, Ballantyne CM, Hsia JA, Kramer JH (2001) Achieving and maintaining national cholesterol education program low-density lipoprotein cholesterol goals with five statins. Am J Med 111: 185191.

Assmann G, Gotto AM Jr (2004) HDL cholesterol and protective factors in atherosclerosis. Circulation 109: III8-III14.

Assmann G, Schriewer H, Schmitz G, Hagele EO (1983). Quantification of HDL-C by precipitation with phosphotungstic acid and $\mathrm{MgCl}$. Clin Chem 29: 2026-2030.

Baigent C, Keech A, Kearney PM, Blackwell L, Buck G, Pollicino C, Kirby A, Sourjina T., Peto R, Collins R et al. (2005) Cholesterol Treatment Trialists' (CTT) Collaborators. Efficacy and safety of cholesterol-lowering treatment: prospective meta-analysis of data from 90,056 participants in 14 randomised trials of statins. Lancet 366: 1267-1278.

Barter P, Gotto AM, LaRosa JC, Moroni J, Szarek M, Grundy SM, Kastelein JJ, Bittner V, Fruchart JC (2007) HDL-cholesterol, very low level of LDL cholesterol and cardiovascular events. $N$ Engl J Med 357: 1301-1310.

Bea F, Blessing E, Bennett B, Levitz M, Wallace EP, Rosenfeld ME (2002) Simvastatin promotes atherosclerotic plaque stability in apoE-deficient mice independently of lipid lowering. Arterioscler Thromb Vasc Biol 22: 1832-1837.

Bela AF, Collins D, Cupplesm LA, Demissie S, Horvath KV, Bloomfield HE, Robins SJ, Schaefer EJ (2009) Value of high-density lipoprotein (HDL) subpopulations in predicting recurrent cardiovascular events in the Veterans Affairs HDL Intervention Trial. Arterioscler Thromb V asc Biol 25: 2185-2191.

Brown WV (2001): Novel approaches to lipid lowering: what is on the horizon. Am J Cardiol 87: 23B-27B.

Bruce C, Tall AR (1995) Cholesteryl ester transfer proteins,reverse cholesterol transport, and atherosclerosis. Curr Opin Lipidol 1995; 6.

Buccolo G, David H (1973) Quantitative determination of serum triglyceride by the use of enzymes. Clin Chem 19: 476-482.

Choudhury RP, Carrelli AL, Stern JD, Chereshnev I, Soccio R, Elmalem VI, Fallon JT, Fisher EA, Reis ED (2004) Effects of simvastatin on plasma lipoproteins and response to arterial injury in wild-type and apolipoprotein-Edeficient mice. I V asc Res 41: 75-83.

Curtiss LK, Plow EF (1984) Interaction of plasma lipoproteins with human platelets. Blood 64: 365-374.

Downs JR, Clearfield M, Weis S (1998) Primary prevention of acute coronary events with lovastatin in men and women with average cholesterol levels: results of AFCAPS/TexCAPS. Air Force/Texas Coronary Atherosclerosis Prevention Study. J Am Med Ass 279: 1615-1622.

Endo A, Tsujita Y, Kuroda M, Tanzawa K (1977) Inhibition of cholesterol synthesis in vitro and in vivo by ML-236A and ML-236B, competitive inhibitors of 3-hydroxy-3-methylglutaryl-coenzyme A reductase. Eur J Biochem 77: 31-36.

Fleisher LN, Tall AR, Witte LD et al. (1982) Stimulation of arterial endothelial cell prostacyclin synthesis by high density lipoproteins. $J$ Biol Chem 257: 6653-6655.

Francone OL, Fielding CJ (1991) Effects of site-directed mutagenesis at residues cysteine-31 and cysteine-184 on lecithin-cholesterol acyltransferase activity. Proc Natl Acad Sci USA 88: 1716-1720.

Friedewald WT, Levy RI, Fredrickson DS (1972) Estimation of the concentration of low-density lipoprotein cholesterol in plasma, without use of the preparative ultracentrifuge. Clin Chem 18: 499502.

Galle J, Ochslen M, Schollmeyer P et al. (1994) Oxidized lipoproteins inhibit endothelium-dependent vasodilation. Effects of pressure and high density lipoprotein. Hypertension 23: 556-564.

Gordon T, Castelli WP, Hjortland MC (1977): High density lipoprotein asa protective factor against coronary heart disease. The Framingham Study. Am J Med 62: 707-714.

Gtez HG, Hovingh GK, Hutten BA, Holleboom AG, Petersen W, Rol P, Stalenhoef A, Zwinderman AH, Groot E, Kastelein JJP, Kuiv- 
enhoven JA (2009) Compromised LCAT function is associated with increased atherosclerosis. Circulation 112: 879-884.

Guohua Song, Jia Liu, Zhenmei Zhao, Yang Yu, Hua Tian, Shutong Yao, Guoli Li, Shucun Qin. (2011) Simvastatin reduces atherogenesis and promotes the expression of hepatic genes associated with reverse cholesterol transport in apoE-knockout mice fed high-fat diet. Lipids Health Disease 10: 8.

Guohua S, Taggart F, Jones P (2011) Effects of statins on high-density lipoproteins: a potential contribution to cardiovascular benefit. Cardiovasc Drugs Ther 22: 321-338.

Jones PH, Davidson MH, Stein EA, Bays HE, McKenney JM, Miller E, Cain VA, Blasetto JW (2003) Comparison of efficacy and safety of rosuvastatin versus atorvastatin, simvastatin and pravastatin across doses (STELLAR* Trial). Am J Cardiol 92: 152-160.

Jones PH, Kafonek S, Laurora I, Hunnunghake D (1998) Comparative dose efficacy study of atorvastatin versus simvastatin, pravastatin, lovastatin and fluvastatin in patients with hypercholesterolemia. Am J Cardiol 81: 582-587.

Koller E, Koller F, Doleschel W (1982) Specific binding sites on human blood platelets for plasma lipoproteins. Hoppe Seylers Z Physiol Chem 363: 395-405.

Mackness MI, Durrington PN, Mackness B (2000) How high-density lipoprotein protects against the effects of lipid peroxidation. Curr Opin Lipidol 11: 383-388.

Masunori M, Toru K, Hiroshi M, Yuji M, Noriaki N, Shinichi O, Yasushi S, Jun S, Kazuaki S, Hiroshige I, and the J-LIT Study Group (2002) Large scale cohort study of the relationship between serum cholesterol concentration and coronary events with low-dose simvastatin therapy in Japanese patients with hypercholesterolemia. Circ J 66: 1087-1095.

Matsuda Y, Hirata K, Inoue N et al. (1993) High density lipoprotein reverses inhibitory effect of oxidized low density lipoprotein on endothelium dependent arterial relaxation. Circ Res 72: 1103-1109.

Nandagopal A, Jangala VR, Subramaniyam K, Srujitpal R, Eashwar R (2008) Study of interaction between antiobesity and hypolipidemic drugs. Int J Health Res 1: 189-195 (e144p26-33).

Nofer JR, Walter M, Kehrel B et al. (1998) HDL3-mediated inhibition of thrombin-induced platelet aggregation and fibrinogen binding occurs via decreased production of phosphoinositide-derived second messengers1,2-diacylglycerol and inositol 1,4,5-tris-phosphate. Arterioscler Thromb Vasc Biol 18: 861-869.
Packman CH, Rosenfeld SI, Leddy JP (1985) High-density lipoprotein and its apolipoproteins inhibit cytolytic activity of complement. Studies onthe nature of inhibitory moiety. Biochim Biophys Acta 812: 107-115.

Rosenfeld SI, Packman CH, Leddy JP (1983) Inhibition of the lytic action of cell-bound terminal complement components by human high density lipoproteins and apoproteins. J Clin Invest 71: 795-808.

Sacks FM, Pfeffer MA, Moye LA. (1996) For the cholesterol and recurrent events trial investigators. The effect of pravastatin on coronary events after myocardial infarction in patients with average cholesterol levels. N Engl J Med 335: 1001-1009.

Shepherd J, Cobbe SM, Ford I (1995) For the West of Scotland Coronary Prevention Study Group. Prevention of coronary heart disease with pravastatin in men with hypercholesterolemia. N Engl J Med 333: 1301-1307.

Shinde S, Chivate N, Kulkarni P, Naikwade N (2013) Hypolipidemic activity of psidium guajava linn leaves extracts in hyperlipidemic rats. Int J Pharm Pharmac Sci 5: 70-72.

Sipahi I, Nicholls SJ, Tuzcu EM, Nissen SE (2006) Coronary atherosclerosis can regress with very intensive statin therapy. Cleve Clin J Med 73: 937-944.

Sposito AC, Chapman MJ (2002) Statin therapy in acute coronary syndromes: mechanistic insight into clinical benefit. Arterioscler Thromb Vasc Biol 22: 1524-1534.

Sugiyama S, Kugiyama K, Matsumura T et al. (1995) Lipoproteins regulate C-type natriuretic peptide secretion from cultured vascular endothelial cells. Arterioscler Thromb Vasc Biol 15: 1968-1974.

Vakeva A, Jauhiainen M, Ehnholm C et al. (1994) High-density lipoproteins can act as carriers of glycophosphoinositol lipid-anchored CD59 in human plasma. Immunology 82: 28-33.

Wang J, Gebre AK, Anderson RA, Parks JS (1997) Cloning and in vitro expression of rat lecithin:cholesterol acyltransferase. Biochem Biophs Acta 1346: 207-211.

Wang YX, Martin-McNulty B, Huw LY, da Cunha V, Post J, Hinchman J, Vergona R, Sullivan ME, Dole W, Kauser K (2002) Antiatherosclerotic effect of simvastatin depends on the presence of apolipoprotein E. Atherosclerosis 162: 23-31.

Zeiher AM, Schachlinger V, Hohnloser SH et al. (1994) Coronary atherosclerotic wall thickening and vascular reactivity in humans. Elevated high-density lipoprotein levels ameliorate abnormal vasoconstriction in early atherosclerosis. Circulation 89: 2525-2532. 